\title{
Characterization of multicopy suppressor genes that complement a defect in the Wis1-Sty1 MAP kinase cascade involved in stress responses in Schizosaccharomyces pombe
}

\author{
Hisami Yamada, Ryusuke Ohmiya, Eiji Yamamoto, Hirofumi Aiba, and Takeshi Mizuno* \\ Laboratory of Molecular Microbiology, School of Agriculture, Nagoya University, Chikusa-ku, Nagoya 464, Japan
}

(Received June 27, 1997; Accepted August 15, 1997)

\begin{abstract}
The Wis1-Sty1 mitogen-activated protein (MAP) kinase cascade is one of the major signaling systems involved in a wide range of stress responses in Schizosaccharomyces pombe. It is known that $\Delta$ wis 1 and $\Delta s t y 1$ mutants exhibit highly pleiotropic phenotypes, including a phenotype of temperature sensitivity for growth. In this study, we screened multicopy suppressor genes that allow both the $\Delta$ wis 1 and $\Delta s t y 1$ mutants to grow simultaneously at a non-permissive temperature, $37^{\circ} \mathrm{C}$. Two such multicopy suppressors were cloned and characterized as sds23+ and $\mathrm{hxk2}^{+}$genes. The former is known to specify a protein that functions as a multicopy suppressor for mutations of the PP1 protein phosphatase and the 20S cyclosome/anaphase-promoting complex (APC), and the latter encodes hexokinase 2. It was revealed that the multicopy sds23+ ${ }^{+}$gene restored a defect in the mating efficiency caused by the $\Delta$ wis 1 and $\Delta s t y 1$ mutations, whereas the multicopy $h \times k 2^{+}$gene suppressed a phenotype of heat-shock sensitivity for growth of these mutant cells. These findings are discussed with special reference to the Wis1-Sty1 MAP kinase signaling pathway in S. pombe.
\end{abstract}

Key Words__ MAP kinase cascade; multicopy suppressor; Schizosaccharomyces pombe; stress response

The exposure of microorganisms to high environmental osmolarity leads to dehydration and a decrease in cell viability. Accordingly, the ability of cells to adapt to external osmotic stress is a fundamental biological process that protects the organisms against fluctuations in water activity and solute content of their environment. In fact, many types of microorganisms, including both prokaryotic and eukaryotic cells, have developed mechanisms to adapt to severe osmotic changes of their environment (this type of adaptive response is often called osmoregulation) (for reviews, see Blomberg and Adler, 1992; Csonka and Hanson, 1991). Although a large number of physiological phenomena related to osmoregulation have been described for many organisms, very little is known about its genetic and molecular bases. However, with special reference to the molecular mechanisms underlying signal transduction, such osmoregulation has recently attracted much attention for both prokaryotes and eukaryotes.

${ }^{*}$ Address reprint requests to: Dr. Takeshi Mizuno, Laboratory of Molecular Microbiology, School of Agriculture, Nagoya University, Chikusa-ku, Nagoya 464, Japan.
The fission yeast Schizosaccharomyces pombe is an organism of choice to gain insight at the molecular level into signal transduction in response to an external osmotic stimulus (or stress) (Millar et al., 1995; Shiozaki and Russell, 1995). In general, the accumulation of osmoprotective compatible solutes inside cells up to the concentrations necessary to counteract the elevation of external osmolarity is a well-documented fact of osmoregulation (Csonka and Hanson, 1991). In S. pombe, glycerol appears to be the major compatible solute, which is synthesized from glycolytic intermediate dihydroxyacetonephosphate in two steps that are catalyzed by an NADH-dependent glycerol-3phosphate dehydrogenase (GPD) and a phosphatase (Blomberg and Adler, 1992). We have cloned the gpd $1^{+}$gene, encoding an osmo-inducible GPD, which was demonstrated to be crucially responsible for osmoregulation in S. pombe (Aiba et al., 1995; Ohmiya et al., 1995, 1997; Yamada et al., 1996). In fact, an insertional inactivation mutant (gpd1:: ura $4^{+}$) shows a severe phenotype of osmosensitivity for growth.

Recent extensive studies of $S$. pombe have begun to shed light on the stress-activated signal transduction mechanism by which $g p d 1^{+}$is activated in re- 
sponse to external high osmolarity (Degols et al., 1996; Degols and Russell, 1997; Kato et al., 1996; Shieh et al., 1997; Shiozaki and Russell, 1996; Wilkinson et al., 1996). First, a mitogen-activated protein (MAP) kinase cascade was found to be involved in this osmosensing signal transduction. The uncovered central elements of this particular MAP-cascade are the Sty1 MAP kinase (also known as Spc1 and Phh1), the Wis1 MAP kinase kinase (MEK), and Wak1 MAP kinase kinase kinase (MEKK), as schematically summarized in Fig. 1. The Sty1 activity is also regulated negatively by Pyp1 and Pyp2 tyrosine-specific phosphatases through direct dephosphorylation of the tyrosine residue of Sty 1 phosphorylated by Wis1. Recent studies have further uncovered a basic-leucine zipper (bZIP) type of transcription factor, Atf1; the phosphorylation (and activation) of which is directly catalyzed by Sty1. Importantly, the osmo-inducible transcription of

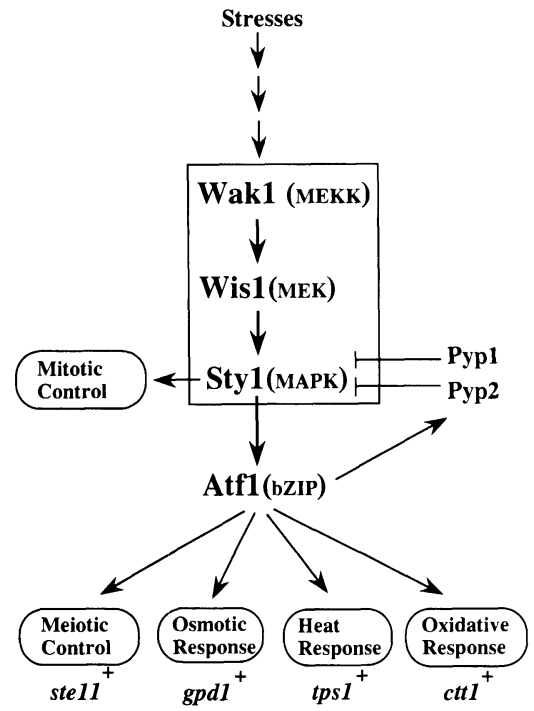

Fig. 1. Schematic representation of the stress-activated Sty1 MAP kinase cascade in S. pombe.

For details, see the text. gpd $1^{+}$is greatly reduced either in wis 1 , sty 1 or atf1 mutants. Consequently, these mutants as well as gpd1 mutants exhibit a phenotype of osmosensitivity for growth.

It should be noted that the Sty 1 MAPK of S. pombe resembles closely the stress-activated kinases of the JNK-p38 (or SAPK-CSBP1) families in mammalian cells (Ziff, 1990). In fact, both the Sty1 and JNK-p38 MAPKs are activated not only by an osmotic stress but also by a range of environmental stresses including nutrient limitation, heat shock, oxidative stress, etc. (see Fig. 1). In this context, $\Delta$ sty 1 or $\Delta$ wis 1 mutants exhibit highly pleiotropic phenotypes, namely, osmosensitive, temperature sensitive, heat sensitive, $\mathrm{H}_{2} \mathrm{O}_{2}$ sensitive, etc. Interestingly, furthermore, the Sty1 MAPK cascade in $S$. pombe is also closely linked to the control of both mitotic and meiotic cell cycle progression (see Fig. 1).

As mentioned above, we have been extensively studying the osmoregulation and Sty 1 MAPK signaling pathway in S. pombe (Aiba et al., 1995; Ohmiya et al., 1995, 1997; Yamada et al., 1996). In this study, to gain new insight into these problems, we attempted to isolate novel types of multicopy suppressor genes which can suppress the temperature sensitive phenotype of both the $\Delta s t y 1$ and $\Delta$ wis 1 mutants simultaneously, in the hope of finding new genes that may be implicated in the Sty1 MAPK signaling cascade and/or GPD1 osmoregulation, because this particular type of approach has not yet been attempted. Here, we characterized two such $S$. pombe genes which were isolated during the attempt.

\section{Materials and Methods}

Strains, plasmids and media. The S. pombe strains used in this study are listed in Table 1. These strains were grown either in YPD medium composed of $1 \%$ yeast extract, $2 \%$ polypeptone and $2 \%$ glucose,

Table 1. List of $S$. pombe strains and plasmids relevant to this study.

\begin{tabular}{|c|c|c|}
\hline Strains/plasmids & Genotype/relevant remarks & Source \\
\hline JY333 & $h^{-}$leu1 ade6-M216 & Y. Imai \\
\hline JM544 & $h^{-}$leu1 ura4-D18 wis $1::$ ura4 $^{+}$ & P. Russell \\
\hline JM1160 & $h^{-}$leu1 ade6-M216 ura4-D18 sty1:: ura4 $^{+}$ & J. Millar \\
\hline JY879 & $h^{90}$ leu1 ade6-M210 ura4 & M. Yamamoto \\
\hline JY808 & $h^{90}$ leu1 ade6-M210 (JY879×JY333) & This study \\
\hline JY809 & $h^{90}$ leu1 ade6-M210 ura4 wis:: ura4 $^{+}(\mathrm{JY} 879 \times \mathrm{JM} 544)$ & This study \\
\hline JY811 & $h^{90}$ leu1 ade6-M210 ura4 sty1:: ura4 ${ }^{+}(\mathrm{JY} 879 \times \mathrm{JM} 1160)$ & This study \\
\hline pDblet & marker $\left(\right.$ ura $\left.^{+}\right)$ & J. A. Huberman \\
\hline pLBDblet & marker $\left(\right.$ leu2 $\left.2^{+}\right)$a derivative of pDblet & This study \\
\hline pLBDblet-GPD & gpd $1^{+}$on pLBDblet & This study \\
\hline pWIS1 & wis $1^{+}$on pLBDblet & This study \\
\hline pSTY1 & sty $1^{+}$on pLBDblet & This study \\
\hline
\end{tabular}


or in SD medium composed of $0.67 \%$ yeast nitrogen base without amino acids (Difco) and supplemented with $2 \%$ glucose and other necessary growth requirements in standard amounts. EMM minimal medium was also used (Moreno et al., 1991). Plasmids used are also listed in Table 1. Plasmid pLBDblet was used as the vector for construction of an S. pombe genomic library.

Recombinant DNA techniques. Conventional recombinant DNA techniques were carried out according to a standard laboratory manual (Sambrook et al., 1989).

Yeast genetics. The procedures used for $S$. pombe genetics in this study are those listed in a standard manual (Moreno et al., 1991).

Assay for mating efficiency. Homotalic haploid cells $\left(h^{90}\right)$ were grown in EMM to $4 \times 10^{6} \mathrm{cells} / \mathrm{ml}$ at $30^{\circ} \mathrm{C}$. Cells were washed with and reinoculated in nitrogen-free minimal medium (EMM-N) at a density of $8 \times 10^{6} \mathrm{celll} / \mathrm{ml}$ and further incubated for $48 \mathrm{~h}$ at $30^{\circ} \mathrm{C}$. Aliquots were taken, and after gentle sonication, the number of cells, zygotes and spores were counted under a microscope. The percentage of mating frequencies was calculated by dividing the number of zygotes plus spores by the number of total cells (one zygote counted as two cells and one spore counted as a half cell).

Heat shock. Cells were grown in SD at $30^{\circ} \mathrm{C}$ for $24 \mathrm{~h}$, reinoculated in fresh SD at $1 \times 10^{6} \mathrm{cells} / \mathrm{ml}$, and grown to $4 \times 10^{6} \mathrm{cells} / \mathrm{ml}$. Aliquots were taken and incubated at $45^{\circ} \mathrm{C}$ for varying times. Cells were diluted and plated onto SD agar plates for incubation at $30^{\circ} \mathrm{C}$. After 3 days-incubation, the number of colonies was counted.

$\mathrm{H}_{2} \mathrm{O}_{2}$ treatment. Cells were grown in SD to $3 \times$ $10^{6} \mathrm{cell} / \mathrm{s} / \mathrm{ml}$ at $30^{\circ} \mathrm{C}$, reinoculated in fresh $\mathrm{SD}$ at $1 \times$ $10^{6} \mathrm{cell} / \mathrm{s} / \mathrm{ml}$, and grown to $4 \times 10^{6} \mathrm{cells} / \mathrm{ml}$. $\mathrm{H}_{2} \mathrm{O}_{2}$ was then added at varying concentrations. The incubation was prolonged for $1 \mathrm{~h}$ at $30^{\circ} \mathrm{C}$. Cells were diluted and plated onto SD agar plates for incubation at $30^{\circ} \mathrm{C}$. After 3 days-incubation, the number of colonies was counted.

\section{Results and Discussion}

Isolation of multicopy suppressor genes for both $\Delta$ sty 1 and $\Delta$ wis 1 mutants

Certain S. pombe mutants, sty $1::$ ura $^{+}$and wis $1:$ : $\mathrm{ura}^{+}{ }^{+}$(or $\Delta$ wis 1 and $\Delta s t y 1$ ), exhibit osmosensitivity for growth as mentioned above. They can grow well on standard YPD and SD plates, but are unable to do so on plates containing $2 \mathrm{M}$ sorbitol. Similarly, these mutants also exhibit temperature sensitivity for growth. They are not capable of forming colonies on standard plates at temperatures higher than $36^{\circ} \mathrm{C}$. In the hope

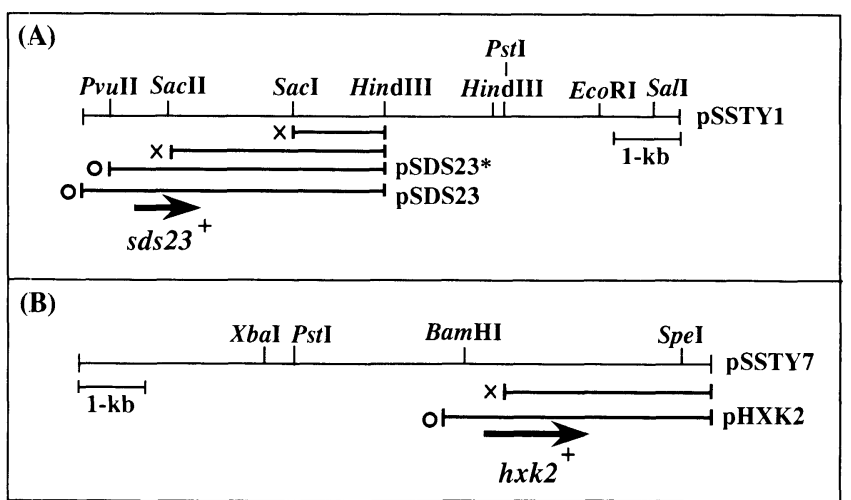

Fig. 2. Schematic representation of the cloned DNA segments from $S$. pombe which suppressed the phenotype of temperature sensitivity for growth of the $\Delta$ wis 1 and $\Delta$ sty 1 mutants.

Plasmids pSSTY1 (panel A) and pSSTY7 (panel B) correspond to the original clones, respectively. Several subclones were isolated, each of which encompasses the indicated DNA region. If it exhibited the ability to suppress, then marked by $O$, otherwise marked by $\times$. Plasmids pSDS23 (or pSDS23*), carrying the $s d s 23^{+}$ gene, and pHXK2, carrying the $h \times k 2^{+}$gene, were mainly used in this study.

of finding $S$. pombe genes that may be relevant to the Sty1 MAPK signaling cascade and/or GPD1 osmoregulation, we screened an $S$. pombe genomic DNA library to look for such multicopy clones on plasmid that simultaneously allows both $\Delta$ sty 1 and $\Delta$ wis 1 mutants to grow at $37^{\circ} \mathrm{C}$ on SD plates. First, we used the $\Delta$ sty 1 mutant as the host to screen the plasmid library and succeeded in isolating several apparently desirable clones. Among them, the clone of sty $1^{+}$itself should be included, and it was confirmed that this was indeed the case. The remainders were further introduced into the $\Delta$ wis 1 mutant to see whether or not they could suppress its temperature sensitivity for growth at $37^{\circ} \mathrm{C}$. Although most of them could not do so, we selected three desirable plasmid clones, each of which presumably carried an $S$. pombe genomic DNA of 8.1$\mathrm{kb}, 9.5-\mathrm{kb}$ and $10.8-\mathrm{kb}$, respectively. These insert DNAs were subjected to extensive analyses by means of restriction endonuclease mapping (Fig. 2). Two of them (i.e., $8.1-\mathrm{kb}$ and $10.8-\mathrm{kb}$ ) were found to carry an overlapping segment; thus, these plasmids (named pSSTY7 and pSSTY1) were selected for further analyses. The restriction maps determined for these two plasmids are shown in Fig. 2.

Identification of the genes responsible for the multicopy suppression of $\Delta$ sty 1 and $\Delta$ wis 1

Based on the results of restriction mapping (Fig. 2), subclones were constructed on the plasmids, and then their abilities to suppress the $\Delta s t y 1$ and $\Delta$ wis 1 mutations were examined in terms of the temperature sensitivity for growth in order to clarify the region responsible for suppression. In one case, certain sub- 
clones exhibited suppression ability but others did not, as shown in Fig. 2A. The $5^{\prime}$-ends of these cloned DNA segments were analyzed by DNA sequencing. These results, together with those from the current nucleotide sequence databases, showed unambiguously that the previously identified $s d s 23^{+}$gene (Ishii et al., 1996) is located in the essential region in the subclones (named pSDS23 and pSDS23*) (Fig. 2A). In another case (Fig. 2B), essentially the same analyses as those described above were carried out. It was found that the previously identified $h \times k 2^{+}$gene (Petit et al., 1996) is located within the region crucial for suppression ability, which is located in plasmid pHXK2 (Fig. 2B).

As demonstrated in Fig. 3, both the plasmids, pSDS23 (or pSDS23*) carrying the sds23 ${ }^{+}$gene and pHXK2 carrying the $h \times k 2^{+}$gene, can respectively exhibit the ability to suppress temperature sensitivity for growth in the $\Delta s t y 1$ and $\Delta$ wis 1 mutants. The results showed that each clone on the plasmids is capable of suppressing these mutations simultaneously, albeit with slightly less efficiency, as compared to the case of the plasmid carrying one each of the authentic genes, sty $1^{+}$or wis $1^{+}$. For example, the sty 1 mutant carrying either the multicopy $s d s 23^{+}$or $h x k 2^{+}$gene was capable of forming colonies on SD medium at $37^{\circ} \mathrm{C}$ after incubation for 4 or 5 days, respectively, while the sty 1 mutant alone could not do so even after incubation for 8 days (data not shown). We concluded therefore that both previously known genes, sds $23^{+}$ and $h \times k 2^{+}$, can somehow function as a multicopy suppressor for the $\Delta$ sty 1 and $\Delta$ wis 1 mutants as far as temperature sensitivity for growth is concerned.

\section{sds $23^{+}$and $h x k 2^{+}$genes and phenotype of osmosen- sitivity}

The $s d s 23^{+}$gene was identified originally as a multicopy suppressor for mutations of $S$. pombe protein phosphatase 1 (PP1) and 205 cyclosome/anaphasepromoting complex (APC), both of which are implicated in the progression from metaphase to anaphase (i.e., onset of anaphase) in the mitotic cell cycle (Ishii

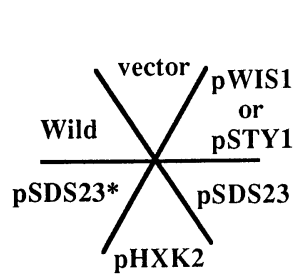

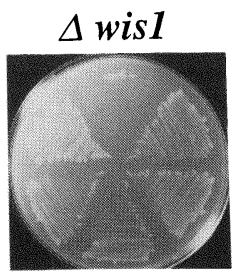

$37^{\circ} \mathrm{C}$

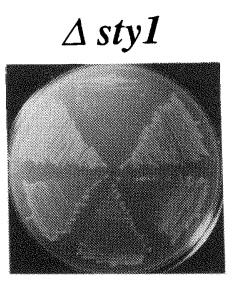

$37^{\circ} \mathrm{C}$
Fig. 3. Examination of temperature sensitivity for growth.

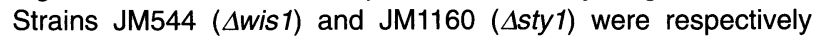
transformed by each indicated plasmid, whereas JY333 was used as the wild-type. These cells were streaked on SD agar plates, and then incubated at $37^{\circ} \mathrm{C}$ for 5 days. et al., 1996). The $h x k 2^{+}$gene was also identified recently as the gene encoding an isozyme of hexokinase (Petit et al., 1996). Until now, no linkage between the $s d s 23^{+}$and $h \times k 2^{+}$genes and the Sty1 MAPK cascade has been reported. However, our results suggest that there appears to be a link between them, directly or indirectly. We then determined whether or not the multicopy $s d s 23^{+}$and $h \times k 2^{+}$genes could suppress other pleiotropic phenotypes of the $\Delta$ wis 1 and $\Delta$ sty 1 mutants. First of all, we addressed this issue in terms of osmosensitivity. Neither of the mutants could form colonies on SD plates containing $2 \mathrm{~m}$ sorbitol (Fig. 4). Even when either of the plasmids carrying $s d s 23^{+}$or $h \times k 2^{+}$was introduced into these mutants, no ability of multicopy suppression was observed, while the plasmids carrying each corresponding authentic gene (wis $1^{+}$or sty $1^{+}$) exhibited the complementation ability under the conditions tested.

\section{sds2 $3^{+}$and $h x k 2^{+}$genes and onset of meiosis}

The Sty1 MAPK cascade appears to be linked to the mitotic G2-M cell cycle control mechanism, thereby resulting in both the $\Delta$ wis 1 and $\Delta$ sty 1 mutants exhibiting a moderate delay of the onset of mitosis, showing an elongated morphology. The Sty 1 MAPK cascade also appears to be linked to the mechanism underlying the onset of meiosis as well, thereby resulting in both mutants exhibiting a greatly reduced level of spore formation (or mating efficiency) upon nitrogen limitation. Thus, the multicopy effects of $s d s 23^{+}$and $h \times k 2^{+}$genes on such cell cycle control were examined for both mutants.

Both $\Delta$ wis 1 and $\Delta s t y 1$ mutants exhibited an elongated cell size when examined and compared with
(A)

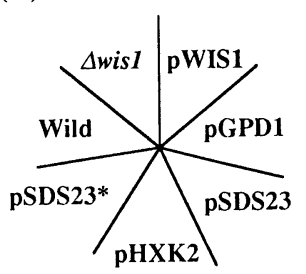

(B)

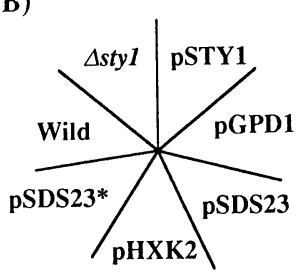

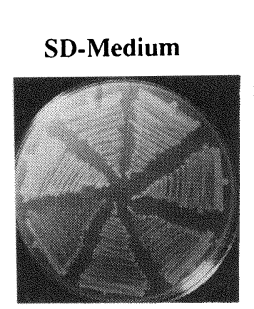

SD-Medium

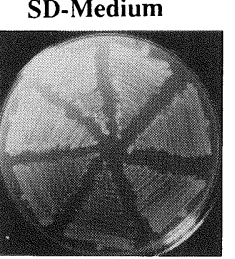

SD-Medium +2 M Sorbitol

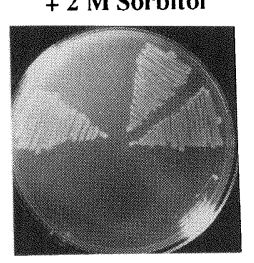

SD-Medium +2 M Sorbitol

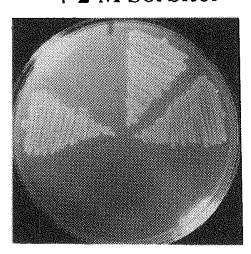

Fig. 4. Examination of osmosensitivity for growth.

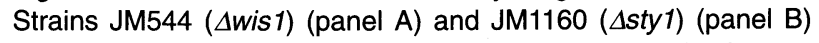
were respectively transformed by each indicated plasmid, whereas JY333 was used as the wild-type. These cells were streaked on SD agar plates and SD agar plates supplemented with $2 \mathrm{M}$ sorbitol, and then incubated at $30^{\circ} \mathrm{C}$ for 5 days. 
wild-type cells under a microscope, as has been reported previously (Shiozaki and Russell, 1995; Warbrick and Fantes, 1991). This phenotype was proposed to be indicative of a moderate delay of the onset of mitosis (particularly, G2-M progression). This particular phenotype was not complemented by the multicopy sds $23^{+}$and $h \times k 2^{+}$genes (data not shown). Thus, the effects of these multicopy genes may not be relevant to the Sty1 MAPK cascade-dependent mitotic control mechanism. We then focused our concern on the well-documented defect of the $\Delta$ wis 1 and $\Delta$ sty 1 mutants in the onset of meiosis. To do so, certain homothallic strains $\left(h^{90}\right)$, each carrying either the wis $1:$ : ura $^{+}$or sty $1::$ ura $^{+}$allele, were first constructed. Both resultant $h^{90} \Delta$ wis 1 and $h^{90} \Delta s t y 1$ cells were confirmed to be sterile (Fig. 5). Upon nitrogen starvation, up to $65 \%$ of $h^{90}$ wild-type cells were able to conjugate, while only $11 \%$ of $h^{90} \Delta$ wis 1 and $10 \%$ of $h^{90} \Delta$ sty 1 cells could do so. It was found here that the multicopy sds $23^{+}$gene is capable of suppressing, at least partially, this sterility for both the mutants. The $h \times k 2^{+}$ gene failed to do so. It was thus suggested that the sds $23^{+}$gene appears to have a special linkage to the Sty 1 MAPK cascade-dependent control of the onset of meiosis, but the $h \times k 2^{+}$gene does not.

\section{sds $23^{+}$and $h \times k 2^{+}$genes and oxidative stress}

It is also known that both $\Delta$ wis 1 and $\Delta s t y 1$ mutant cells are hypersensitive to oxidative stress such as $\mathrm{H}_{2} \mathrm{O}_{2}$ treatment. When rapidly growing mutant cells were treated with $\mathrm{H}_{2} \mathrm{O}_{2}$ in $\mathrm{SD}$ medium at $30^{\circ} \mathrm{C}$ for $1 \mathrm{~h}$, they lost viability much more rapidly than in the case of the wild-type cells (Fig. 6). Neither the $s d s 23^{+}$nor

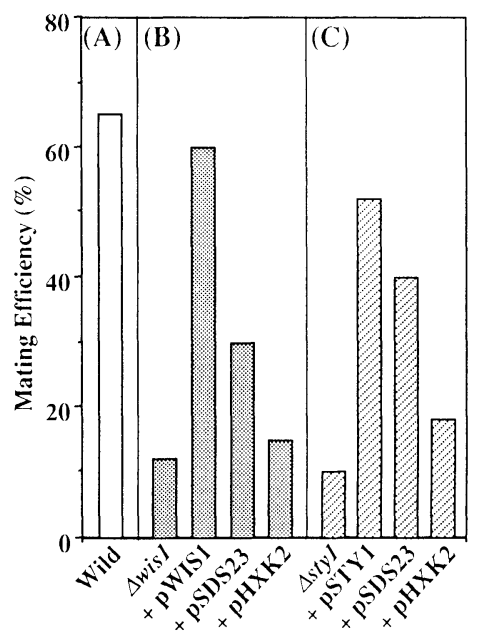

Fig. 5. Examination of mating efficiency.

Homothallic strains JY809 $\left(h^{90} \Delta\right.$ wis1) (panel B) and JY811 $\left(h^{90} \Delta\right.$ sty1) (panel C) were transformed by each indicated plasmid, whereas JY808 $\left(h^{90}\right)$ (panel A) was used as the wild-type. For these cells, mating efficiency was assayed (see MATERIALS AND METHODS). $h \times k 2^{+}$gene is able to suppress this particular phenotype, as shown in Fig. 6.

\section{sds $23^{+}$and $h \times k 2^{+}$genes and phenotype of heat toler- ance}

It has been reported that both $\Delta$ wis 1 and $\Delta s t y 1$ mutant cells are sensitive to heat treatment (or heat shock). When these mutant cells, rapidly growing in SD minimal medium, were exposed to high temperatures (e.g., $45^{\circ} \mathrm{C}$ ) for various amounts of time, they lost viability much more rapidly than in the case of the wild-type cells (Fig. 7). It was found that this particular phenotypic defect of both mutants was suppressed, at least partially, by the introduction of the multicopy $h \times k 2^{+}$gene, while the sds $23^{+}$gene did not show such ability. It was thus suggested that the $h x k 2^{+}$gene appears to have a special linkage to Sty1 MAPK cascade-dependent heat response, but the $s d s 23^{+}$gene does not.
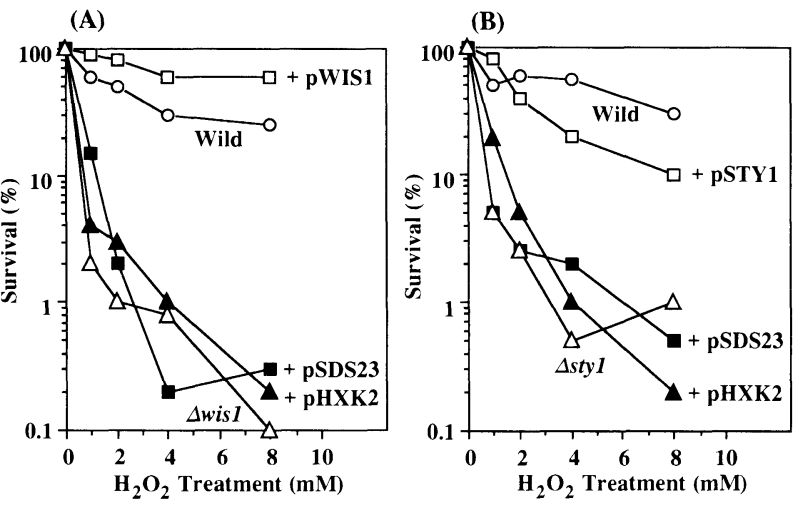

Fig. 6. Examination of viability upon $\mathrm{H}_{2} \mathrm{O}_{2}$ treatment.

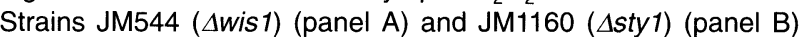
were respectively transformed by each indicated plasmid, whereas JY333 was used as the wild-type. For these cells, viability after $\mathrm{H}_{2} \mathrm{O}_{2}$ treatment was examined (see MATERIALS AND METHODS).
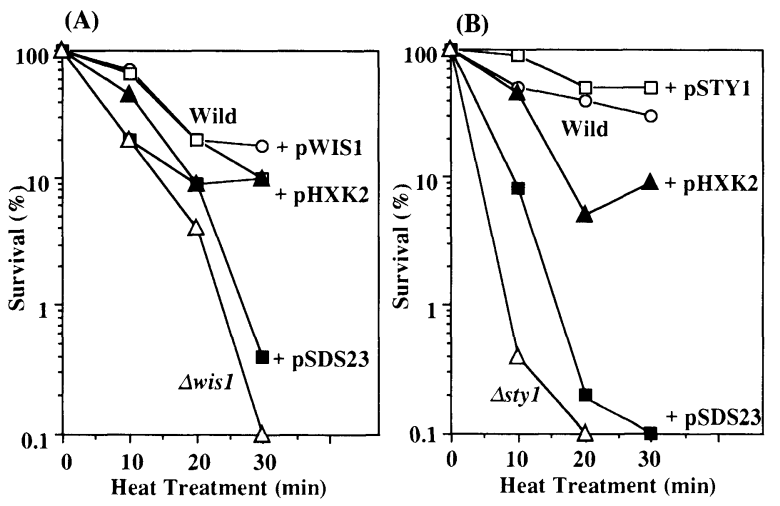

Fig. 7. Examination of viability upon heat treatment.

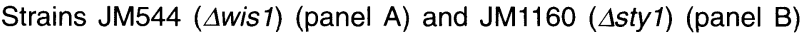
were respectively transformed by each indicated plasmid, whereas JY'333 was used as the wild-type. For these cells, viability upon heat treatment at $45^{\circ} \mathrm{C}$ was examined (see MATERIALS AND METHODS). 


\section{General implication}

In this study, we identified two genes, sds23 $3^{+}$and $h \times k 2^{+}$, both of which are able to suppress the phenotype of temperature sensitivity for growth in both $\Delta$ wis 1 and $\Delta$ sty 1 mutants provided that they were introduced with a multicopy plasmid into the deletion mutants (Fig. 3). These genes are able to suppress, only partially, the pleiotropic phenotypes of the $\Delta$ wis 1 and $\Delta$ sty 1 mutants. In fact, the phenotypes of osmosensitivity and $\mathrm{H}_{2} \mathrm{O}_{2}$ sensitivity for growth did not recover to the level of wild-type cells by the introduction of these multicopy genes (Figs. 4 and 6). Interestingly, however, the multicopy $s d s 23^{+}$gene restored the defect in mating efficiency caused by the mutations (Fig. 5), whereas the multicopy $h x k 2^{+}$gene suppressed the phenotype of heat sensitivity for growth of the mutant cells (Fig. 7). It thus appears to be likely that presumed manners by which Sds23 and Hxk2 proteins interact with the Sty1 MAPK cascade are indirect and different from each other.

The $s d s 23^{+}$gene was originally identified as a multicopy suppressor for mutations of $S$. pombe protein phosphatase 1 (PP1) and 20S cyclosome/APC, which are involved in mitotic cell proliferation (Ishii et al., 1996). They reported that the $s d s 23^{+}$gene is not essential for cell viability, but a mutant (sds23:: ura4 ${ }^{+}$) cannot form colonies at 22 and $36^{\circ} \mathrm{C}$ (i.e., sensitive for growth both at low and high temperatures). In the sds23 deletion mutant, the progression of anaphase and cytokinesis is retarded, and cell shape is aberrant. Thus, Sds23 protein appears to be involved not only in mitotic control but also at least in two other cellular events as well (i.e., cytokinesis and cell-shape control). In this study, we found that Sds23 should also be implicated in another major cellular event, meiotic control (Fig. 5). In any event, the actual molecular function of Sds23 is elusive. In this respect, our observation should give us a new hint to address the issues relevant to the intriguing cellular functions of Sds23. In fact, our preliminary observation showed that an sds23 deletion mutant exhibits a phenotype of osmosensitivity for growth in a manner independent of GPD1 osmoregulation (Ohmiya, R. and Mizuno, T., unpublished result).

In general, hexokinase initiates the metabolism of sugars (i.e., glycolysis) in a variety of organisms. In $S$. pombe, two types of hexokinase have been characterized and their corresponding genes have been cloned, $h \times k 1^{+}$and $h x k 2^{+}$, respectively (Petit et al., 1996). The former encodes hexokinase 1 with a low phosphorylation coefficient on glucose, and the latter encodes hexokinase 2 that is kinetically conventional. Note that the latter is the one identified in this study as the multicopy suppressor for the Sty1 MAPK cascade mutants. As far as the primary sequences are concerned, they present a high overall homology with the hexokinases of Saccaromyces cerevisiae (Hxk1p and Hxk2p): $50 \%$ in the case of $h \times k 1^{+}$and $38 \%$ in the case of $h \times k 2^{+}$. In fact, Hxk2 of $S$. pombe is more similar to the glucokinase of $S$. cerevisiae (Glk1p). It may be worth mentioning that a disruption of the $h \times k 1^{+}$gene in $S$. pombe has no effect on growth, but a disruption of the $h \times k 2^{+}$gene doubles the generation time in glucose. In regard to these $S$. pombe $h x k^{+}$genes, however, nothing more is known about them. In this respect, our intriguing finding is that the multicopy $h x k 2^{+}$gene renders the $\Delta$ wis 1 and $\Delta$ sty 1 mutants heat tolerant.

A current view as to the Sty1 MAPK cascade-dependent mechanism underlying the heat tolerance of $S$. pombe cells is as follows. In S. pombe, an elevated accumulation of trehalose appears to play a major role in heat tolerance (Blazouez et al., 1994; De Virgilio et al., 1990). In fact, the tps $1^{+}$gene encoding trehalose6-phosphate synthase is activated upon heat shock at the level of transcription in a manner dependent largely on the Sty1 MAPK cascade (see Fig. 1) (Degols et al., 1996). However, the induction of $t p s 1^{+}$expression is not completely abolished in $\Delta$ sty 1 cells, suggesting that there may also be an unknown Sty1 MAPK cascade-independent mechanism inducing tps $1^{+}$expression in response to heat stress. Considering the fact that glucose-6-phosphate is the immediate processor of trehalose, it is possible to envisage that an overproduction of Hxk2 presumably catalyzing glucose-6-phosphate synthesis may enhance the Sty1 MAPK cascade-independent pathway of trehalose synthesis, thereby enabling suppression of the defect in Sty1 MAPK cascade-dependent heat tolerance. This naive but plausible idea remains to be elucidated.

In short, the Sty1 MAPK cascade is one of the major signaling systems and responsible for a wide range of major cellular events in $S$. pombe. Clarification of this highly complicated MAPK cascade awaits further extensive experimentation. During the course of our approach, we have isolated a few more multicopy clones which can suppress both $\Delta$ wis 1 and $\Delta$ sty 1 mutations (Yamada, $H$. and Mizuno, T., unpublished result). Characterization of these unidentified clones, together with $s d s 23^{+}$and $h x k 2^{+}$, should give us hints for understanding the complex mechanisms underlying the Sty1 MAPK cascade. These are currently underway.

The authors are grateful to the following doctors for their kind gifts (i.e., strains and plasmids): Y. Imai and M. Yamamoto (The University of Tokyo, Japan), P. Russell (The Scripps Research Institute, U.S.A.), J. B. A. Millar (National Institute for Medical Research, U.K.), J. A. Huberman (Roswell Park Cancer Institute, U.S.A.). This work was supported by Grants-in-Aid for Scientific Research from the Ministry of Education, Science, Sports and Culture of Japan. 


\section{References}

Aiba, H., Yamada, H., Onmiya, R., and Mizuno, T. (1995) The osmo-inducible $g p d 1^{+}$gene is a target of the signaling pathway involving Wis1 MAP-kinase kinase in fission yeast. FEBS Lett., 376, 199-201.

Blazouez, M., Stucka, R., Feldmann, H., and Gancedo, C. (1994) Trehalose-6-P synthase is dispensable for growth on glucose but not for spore germination in Schizosaccharomyces pombe. J. Bacteriol., 176, 3895-3902.

Blomberg, A. and Adler, L. (1992) Physiology of osmotolerance in fungi. Adv. Microbiol. Physiol., 33, 326-329.

Csonka, L. N. and Hanson, A. D. (1991) Prokaryotic osmoregulation, Annu. Rev. Microbiol., 45, 569-606.

De Virgilio, C., Simmen, U., Hottiger, T., Boller, T., and Wiemken, T. B. (1990) Heat shock induces enzymes of trehalose metabolism, trehalose accumulation, and thermotolerance in Schizosaccharomyces pombe, even in the presence of cycloheximide. FEBS Lett., 273, 107-110.

Degols, G. and Russell, P. (1997) Discrete roles of the Spc1 kinase and the Atf1 transcription factor in the UV response of Schizosaccaromyces pombe. Mol. Cell. Biol., 17, 3356-3363.

Degols, G., Shiozaki, K., and Russell, P. (1996) Activation and regulation of the Spc1 stress-activated protein kinase in Schizosaccharomyces pombe. Mol. Cell. Biol., 16, 2870-2877.

Ishii, K., Kumada, K., Yoda, T., and Yanagida, M. (1996) Requirement for PP1 phosphatase and 20S cyclosome/APC for the onset of anaphase is lessened by the dosage increase of a novel gene sds23 ${ }^{+}$. EMBO J., 15, 6629-6640.

Kato, T., Okazaki, K., Murakami, H., Stettler, S., Fantes, P. A., and Okayama, H. (1996) Stress signal, mediated by a Hog1-like MAP kinase, controls sexual development in fission yeast. FEBS Lett., 378, 207-212.

Millar, J. B. A., Buck, V., and Wilkinson, M. G. (1995) Pyp1 and Pyp2 PTPases dephosphorylate an osmosensing MAP kinase controlling cell size at division in fission yeast. Genes Dev., 9, 2117-2130.

Moreno, S., Klar, A., and Nurse, P. (1991) Molecular genetic analysis of fission yeast Schizosaccharomyces pombe. Methods Enzymol., 194, 795-823.

Ohmiya, R., Aiba, H., Yamada, H., and Mizuno, T. (1997) Clarification of the promoter structure of the osmoregulated $g p d 1^{+}$gene encoding an isozyme of NADH-dependent glycerol-3-phosphate dehydrogenase in fission yeast. Biosci. Biotech. Biochem., 61, 553-555.

Onmiya, R., Yamada, H., Nakashima, K., Aiba, H., and Mizuno, T. (1995) Osmoregulation of fission yeast: Cloning of two distinct genes encoding glycerol-3-phosphate dehydrogenase, one of which is responsible for osmotolerance for growth. Mol. Microbiol., 18, 963-973.

Petit, T., Blazquez, A., and Gancedo, C. (1996) Schizosaccaromyces pombe possesses an unusual and a conventional hexokinase: Biochemical and molecular characterization of both hexokinases. FEBS Lett., 378, 185-189.

Sambrook, J., Fritsch, E. F., and Maniatis, T. (1989) Molecular Cloning: A Laboratory Manual, Cold Spring Harbor Laboratory Press, Cold Spring Harbor, New York.

Shieh, J.-C., Wilkinson, M. G., Buck, V., Morgan, B., Makino, K., and Millar, J. B. A. (1997) The Mcs4 response regulator coordinately controls the stress-activated Wak1-Wis1-Sty1 MAP kinase pathway and fission yeast cell cycle. Genes Dev., 11, 1008-1022.

Shiozaki, K. and Russell, P. (1995) Cell-cycle control linked to extracellular environment by MAP kinase pathway in fission yeast Nature, 377, 739-743.

Shiozaki, K. and Russell, P. (1996) Conjugation, meiosis, and the osmotic stress response are regulated by Spc1 kinase through Atf1 transcription factor in fission yeast. Genes Dev., 10, 22762288.

Warbrick, E. and Fantes, P. A. (1991) The Wis1 protein kinase is a dosage-dependent regulator of mitosis in Schizosaccaromyces pombe. EMBO J., 13, 4291-4299.

Wilkinson, M., Samuels, M., Takeda, T., Toone, W. M., Shieh, J.-C., Toda, T., Millar, J. B. A., and Jones, N. (1996) The Atf1 transcription factor is a target for the Sty1 stress-activated MAP kinase pathway in fission yeast. Genes Dev., 10, 2289-2301.

Yamada, H., Ohmiya, R., Aiba, H., and Mizuno, T. (1996) Construction and characterization of a deletion mutant of gpd2 that encodes an isozyme of NADH-dependent glycerol-3-phosphate dehydrogenase in fission yeast. Biosci. Biotech. Biochem., 60, 918-920.

Ziff, E. B. (1990) Transcription factors: A new family gathers at the cAMP response site. Trends Genet., 6, 69-72. 